\title{
Radiative neutrino mass generation and dark energy
}

\author{
K. Bamba, C. Q. Geng and S. H. Ho \\ Department of Physics, National Tsing Hua University, Hsinchu, Taiwan 300
}

(Dated: December 2, 2018)

\begin{abstract}
We study the models with radiative neutrino mass generation and explore the relation between the neutrino masses and dark energy. In these models, the pseudo-Nambu-Goldston bosons (pNGBs) arise at two-loop level via the Majorana neutrino masses. In particular, we demonstrate that the potential energy of the pNGB can be the dark energy potential and the observed value of the equation of state $(\mathrm{EoS})$ parameter of the universe, i.e., $w \simeq-1$, can be realized.

PACS numbers: 98.80.Cq,14.60.Pq,14.80.Cp,14.80.Cp
\end{abstract}




\section{INTRODUCTION}

In the standard model (SM) of particle physics, neutrinos are massless. However, various experimental searches indicate that neutrinos have tiny masses $\left(\leq O\left(10^{-2}\right) \mathrm{eV}\right)[1]$. It is a challenging and important problem to explain the origin of the small neutrino masses. Various mechanisms could generate neutrino masses [2], in which the one with radiative neutrino mass generation without right-handed neutrinos by extending the Higgs sector [3, 4, 5] is particularly interesting because the neutrino masses are naturally small. It is clear that without the right-handed states the active neutrinos can only have Majorana masses.

On the other hand, recent cosmological observations have confirmed that not only there existed the inflationary stage in the early universe, but also at present the expansion of the universe is accelerating [6, 7, 8]. Although various scenarios for the late-time acceleration in the expansion of the universe have been proposed, the cosmic acceleration mechanism is still not well understood [9, 10, 11, 12, 13].

In the framework of general relativity, the current accelerating universe is due to the so-called dark energy (or cosmological constant) with its density at the present time is only about $\left(10^{-3} \mathrm{eV}\right)^{4}$, which is much smaller than any known energy scale in particle physics except the neutrino masses. It is interesting to note that the energy scale of dark energy coincides with the neutrino masses as discussed in Ref. [14].

Recently, it has been suggested [15, 16, 17, 18] that the neutrino masses vary as a function of a scalar field, called the "acceleron", which drives the universe to its present accelerating phase. Such neutrinos are referred as mass varying neutrinos (MaVaNs). The effects of the MaVaNs on the anisotropy of the cosmic microwave background (CMB) radiation and large scale structure (LSS) have been studied in Ref. [19]. Several models with the generation of the MaVaNs through the see-saw mechanism with right-handed neutrinos to account for the baryon asymmetry in the universe have been proposed in Refs. [20, 21]. In these studies, one has to break a global symmetry spontaneously to get a Nambu-Goldston boson (NGB) and introduce a soft symmetry breaking term so that the NGB receives a soft mass via a loop diagram and becomes a pseudo-Nambu-Goldston boson (pNGB). This pNGB corresponds to the acceleron field in the scenario proposed in Ref. [15]. Some models to explain neutrino masses and dark energy at the TeV scale have been explored in Refs. [22, 23]. Moreover, 
the Majorana neutrino superfluidity and the stability of the neutrino dark energy have been discussed in Ref. [24].

In this paper, we consider the generation of the small Majorana neutrino masses through the radiative mechanism without right-handed neutrinos in the framework of the extended Babu-Zee model [4]. Here, we do not introduce a soft breaking term "by hand", but induce one from loop diagrams. In other words, we break global symmetries spontaneously in the first place, and then, via loop diagrams, introduce a soft (original) symmetry breaking term followed by a mass term for the pNGB. This pNGB also plays a role of the acceleron field. As a result, we show that the small neutrino masses depend on the pNGB and we argue that the potential energy of the pNGB can be the potential of dark energy. Furthermore, we demonstrate that the observed value of the equation of state (EoS) parameter from the Wilkinson Microwave Anisotropy Probe (WMAP) data on the anisotropy of the CMB radiation can be realized by following the discussion in Refs. [15, 16, 17].

\section{PSEUDO-NAMBU-GOLDSTON BOSON AS THE ACCELERON FILED}

In the Babu-Zee model [4], it contains only two extra scalar bosons beyond the SM, i.e., one singly charged scalar $\left(h^{+}\right)$and one doubly charged scalar $\left(k^{++}\right)$. In this study, we would extend the Babu-Zee model [4] by considering three singly charged scalars: $h_{e \mu}^{+}, h_{e \tau}^{+}$ and $h_{\mu \tau}^{+}$; and three doubly charged scalars: $k_{e \mu}^{++}, k_{e \tau}^{++}$and $k_{\mu \tau}^{++}$, which carry different lepton numbers. In addition, we have to introduce singlet scalars $\left(\Phi_{a b}\right)$ to break the lepton number symmetries and induce non-zero phases. The particle contents and quantum numbers are shown in Table I, where $U(1)_{e}, U(1)_{\mu}$ and $U(1)_{\tau}$ represent the electron, muon and tau number symmetries, $l_{L}$ and $l_{R}$ are the left-handed lepton doublet and right-handed lepton singlet, $H$ is the Higgs doublet in the SM, and $h_{a b}^{+}$and $k_{a b}^{++}$are singly and doubly charged scalars, respectively.

The Yukawa couplings between the singly and doubly charged scalars and fermions are given by

$$
\mathcal{L}_{\mathcal{Y}}=f_{a b}\left(l_{a L}^{T i} C l_{b L}^{j}\right) \epsilon_{i j} h_{a b}^{+}+g_{a b}\left(l_{a R}^{T} C l_{b R}\right) k_{a b}^{++}+\text {h.c. }
$$

where $C$ is the charge conjugation matrix, $i, j$ and $a, b$ are $S U(2)_{L}$ and generation indices, 
respectively. In our model, it is convenient to expand the Lagrangian in Eq. (11) as follows:

$$
\begin{aligned}
\mathcal{L}_{\mathcal{Y}}= & 2\left[f_{e \mu}\left(\bar{\nu}_{e}^{c} \mu_{L}-\bar{\nu}^{c}{ }_{\mu} e_{L}\right) h_{e \mu}^{+}+f_{e \tau}\left(\bar{\nu}_{e}^{c} \tau_{L}-\bar{\nu}_{\tau}^{c} e_{L}\right) h_{e \tau}^{+}+f_{\mu \tau}\left(\bar{\nu}_{\mu}^{c} \tau_{L}-\bar{\nu}_{\tau}^{c} \mu_{L}\right) h_{\mu \tau}^{+}\right. \\
& \left.+g_{e \mu}\left(\overline{e^{c}} \mu_{R}\right) k_{e \mu}^{++}+g_{e \tau}\left(\overline{e^{c}} \tau_{R}\right) k_{e \tau}^{++}+g_{\mu \tau}\left(\bar{\mu}^{c} \tau_{R}\right) k_{\mu \tau}^{++}\right]+ \text {h.c. },
\end{aligned}
$$

where we have used $f_{a b}=-f_{b a}, g_{a a}=0$ and $g_{c d}=g_{d c}(c \neq d)$. The Higgs potential can be written as two parts:

$$
\begin{gathered}
\mathcal{L}_{1}=\sum_{\xi, \eta} \beta_{\xi}^{\eta}\left(h_{\xi}^{+} h_{\eta}^{+} k_{\eta}^{--} \Phi_{\xi}\right)+\beta_{e e}^{\mu \tau}\left(h_{e \mu}^{+} h_{e \tau}^{+} k_{\mu \tau}^{--} \Phi_{e e}\right)+\beta_{\mu \mu}^{e \tau}\left(h_{e \mu}^{+} h_{\mu \tau}^{+} k_{e \tau}^{--} \Phi_{\mu \mu}\right) \\
+\beta_{\tau \tau}^{e \mu}\left(h_{e \tau}^{+} h_{\mu \tau}^{+} k_{e \mu}^{--} \Phi_{\tau \tau}\right)+\text { h.c. }
\end{gathered}
$$

where $\xi, \eta=e \mu, e \tau, \mu \tau$ and

$$
\begin{aligned}
\mathcal{L}_{2}= & \mu^{2}\left(H H^{\dagger}\right)+\lambda\left(H H^{\dagger}\right)^{2}+\mu_{i j}^{2}\left(h_{i j}^{+} h_{i j}^{-}\right)+\lambda_{i j}\left(h_{i j}^{+} h_{i j}^{-}\right)^{2}+\tilde{\mu}_{i j}^{2}\left(k_{i j}^{++} k_{i j}^{--}\right) \\
& +\tilde{\lambda}_{i j}\left(k_{i j}^{++} k_{i j}^{--}\right)^{2}+\kappa_{i j}\left(H H^{\dagger}\right)\left(h_{i j}^{+} h_{i j}^{-}\right)+\tilde{\kappa}_{i j}\left(H H^{\dagger}\right)\left(k_{i j}^{++} k_{i j}^{--}\right) \\
& +C_{i j l m}\left(h_{i j}^{+} h_{i j}^{-}\right)\left(k_{l m}^{++} k_{l m}^{--}\right)+\tilde{C}_{i j l m}\left(h_{l m}^{+} h_{i j}^{-}\right)\left(k_{i j}^{++} k_{l m}^{--}\right) .
\end{aligned}
$$

We remark that there are enough degrees of freedom to redefine the fields and make all the coefficients in Eqs. (2) and (3) real. We also note that it is not necessary to include all three $\Phi_{a a}(a=e, \mu, \tau)$ to get a realistic model. However, to have a non-vanishing phase field, at least one of them is needed. Furthermore, in the model the lepton symmetries

TABLE I: The particle contents and quantum numbers, where $l_{L}$ and $l_{R}$ are the left-handed lepton doublet and right-handed lepton singlet, $h_{a b}^{-}$and $k_{a b}^{--}$are singly and doubly charged scalars, and $a, b=e, \mu$ and $\tau$.

\begin{tabular}{|c|c|c|}
\hline Particles & $S U(2)_{L} \times U(1)_{Y}$ & $U(1)_{e} \times U(1)_{\mu} \times U(1)_{\tau}$ \\
\hline \hline$l_{L a}$ & $(2,-1)$ & $\left(\delta_{a e}, \delta_{a \mu}, \delta_{a \tau}\right)$ \\
\hline$l_{R a}$ & $(1,-2)$ & $\left(\delta_{a e}, \delta_{a \mu}, \delta_{a \tau}\right)$ \\
\hline \hline$H$ & $(2,1)$ & $(0,0,0)$ \\
\hline$h_{a b}^{-}$ & $(1,-2)$ & $\left(\delta_{a e}+\delta_{b e}, \delta_{a \mu}+\delta_{b \mu}, \delta_{a \tau}+\delta_{b \tau}\right)$ \\
\hline$k_{a b}^{--}$ & $(1,-4)$ & $\left(\delta_{a e}+\delta_{b e}, \delta_{a \mu}+\delta_{b \mu}, \delta_{a \tau}+\delta_{b \tau}\right)$ \\
\hline$\Phi_{a b}$ & $(1,0)$ & $\left(\delta_{a e}+\delta_{b e}, \delta_{a \mu}+\delta_{b \mu}, \delta_{a \tau}+\delta_{b \tau}\right)$ \\
\hline
\end{tabular}


are spontaneously broken after the singlet scalar fields $\Phi_{a b}$ acquire the vacuum expectation values $(\mathrm{VEVs}) v_{a b}$. For convenience, we parametrize $\Phi_{a b}$ as nonlinear $\sigma$ fields in terms of the Nambu-Goldstone bosons (NGBs) $\phi_{a b}$ by

$$
\Phi_{a b}=v_{a b} \exp \left(i \phi_{a b} / v_{a b}\right)
$$

For simplicity, we assume that all VEVs are the same, i.e., $v_{a b}=v$. It is clear that there are only three independent NGB states, defined by

$$
\psi_{1}=\phi_{e \mu}-\phi_{e e} / 2-\phi_{\mu \mu} / 2, \quad \psi_{2}=\phi_{e \tau}-\phi_{e e} / 2-\phi_{\tau \tau} / 2, \quad \psi_{3}=\phi_{\mu \tau}-\phi_{\mu \mu} / 2-\phi_{\tau \tau} / 2 .
$$

\section{NEUTRINO MASSES}

As the original Babu-Zee model [4], the neutrinos receive Majorana masses induced radiatively through the two-loop diagrams shown in Fig. 1. Consequently, the Majorana neutrino mass term is found to be [4, 5, 25, 26]

$$
\begin{aligned}
\mathcal{L}_{\text {mass }} & =-\frac{1}{2}\left(\mathcal{M}_{\nu}\right)_{a b}\left(\overline{\nu_{L}^{c}}\right)_{a}\left(\nu_{L}\right)_{b}+\text { h.c. }, \\
\mathcal{M}_{\nu}= & \left(\begin{array}{ccc}
M_{e e} & M_{e \mu} e^{i \psi_{1} / v} & M_{e \tau} e^{i \psi_{2} / v} \\
M_{\mu e} e^{-i \psi_{1} / v} & M_{\mu \mu} & M_{\mu \tau} e^{i \psi_{3} / v} \\
M_{\tau e} e^{-i \psi_{2} / v} & M_{\mu \tau} e^{-i \psi_{3} / v} & M_{\tau \tau}
\end{array}\right),
\end{aligned}
$$

with $M_{a b}=M_{b a}$ and

$$
M_{a b}=\sum_{c, d} 8 \beta_{a b}^{c d} g_{c d} f_{d a} f_{b c} m_{c} m_{d} v I_{c d}
$$

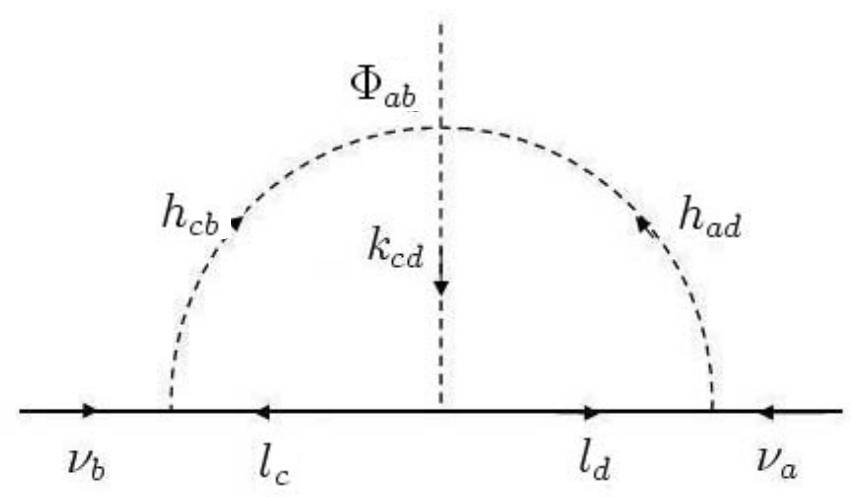

FIG. 1: Two-loop diagrams to generate neutrino masses. 
where we have redefined the neutrinos by

$$
\nu_{a} \exp \left(i \phi_{a a} / 2 v\right) \rightarrow \nu_{a}
$$

$\beta_{a b}^{c d}$ are symmetric under the exchange of $a \leftrightarrow b$ or $c \leftrightarrow d$ and $I_{c d}$ is the loop integral, given by

$$
I_{c d}=\int \frac{d^{4} k}{(2 \pi)^{4}} \frac{d^{4} q}{(2 \pi)^{4}} \frac{1}{\left(k^{2}-m_{c}^{2}\right)} \frac{1}{\left(k^{2}-m_{h_{b d}}^{2}\right)} \frac{1}{\left(q^{2}-m_{d}^{2}\right)} \frac{1}{\left(q^{2}-m_{h_{a c}}^{2}\right)} \frac{1}{\left((k-q)^{2}-m_{k_{c d}}^{2}\right)},
$$

with $m_{c, d}, m_{h_{a c}, h_{b d}}$ and $m_{k_{c d}}$ being the corresponding masses of the charged fermions, singly and doubly charged scalars, respectively. Here, we have assumed that all couplings of $f_{a b}$ and $g_{a b}$ are real. We note that all three NGBs become pNGBs because the induced neutrino masses lead to soft breaking terms into the theory to explicitly break the global symmetries. We remark that, unlike the original Babu-Zee model [4], there only exist few terms contributing to the neutrino masses because of three global $U(1)$ symmetries.

To estimate $M_{a b}$ in Eq. (8), we take $m_{c, d} \ll m_{h_{a c}, h_{b d}} \sim m_{h}$ and $m_{c, d} \ll m_{k_{c d}} \sim m_{k}$. In these approximations, the loop integral in Eq. (10) can be simplified as [25, 26]

$$
I_{c d} \simeq \frac{1}{\left(16 \pi^{2}\right)^{2}} \frac{1}{m_{h}^{2}} \tilde{I}\left(\frac{m_{k}^{2}}{m_{h}^{2}}\right),
$$

where the dimensionless function of $\tilde{I}(x)$ is a smooth function from $\pi^{2} / 3$ decreasing to $\simeq 0.8$ for an interval $10^{-3}<x<10$. In the Babu-Zee model [4] there exist some tree-level lepton number violating processes. In our model, there are no such tree processes because we introduce more charged scalars couple to different "pairs" of leptons. However, these processes could be induced at the loop level, which will be ignored in our present discussions. Furthermore, there is no t-channel $k^{++}$exchange for the muonium-antimuonium oscillation because there are no $\bar{e}_{R}^{c} e_{R} k^{++}$and $\bar{\mu}_{R}^{c} \mu_{R} k^{++}$couplings in our model. Moreover, there are no lepton number violating radiative decays of $\ell_{2} \rightarrow \ell_{1} \gamma\left(\ell_{1} \neq \ell_{2}\right)$ because different pairs of leptons are coupled by different charged scalars. On the other hand, in our model there are contributions to lepton number conserving processes such as $g-2$ and $\ell_{2} \rightarrow \ell_{1} \nu_{2} \bar{\nu}_{2}$, which give some loose constraints on the couplings of $f_{a b}$ and $g_{a b}$ [25]. To illustrate the numerical estimation, by choosing that $\beta_{a b}^{c d} \sim 1, f_{a b} \sim 0.1, g_{a b} \sim 1$, and $m_{h} \sim m_{k} \sim v \sim 1 \mathrm{TeV}$, all neutrino mass elements in Eq. (8) are found to be $\leq O\left(10^{-2} \mathrm{eV}\right)$. We note that since there are too many free parameters in the model, it is always possible to obtain the realistic neutrino mixings. Finally, we remark that the three singlet fields of $\Psi_{a a}(a=e, \mu, \tau)$ can be reduced to one without altering the feature of the model. 


\section{DARK ENERGY}

Similar to Ref. [27] (see also [21]), we can write down the induced potential for pNGBs as follows:

$$
V\left(\psi_{1}, \psi_{2}, \psi_{3}\right) \sim \frac{1}{32 \pi^{2}} \operatorname{Tr}\left[\mathcal{M}_{\nu} \mathcal{M}_{\nu}^{\dagger} \mathcal{M}_{\nu} \mathcal{M}_{\nu}^{\dagger} \ln \frac{\Lambda^{2}}{\mathcal{M}_{\nu} \mathcal{M}_{\nu}^{\dagger}}\right],
$$

where $\Lambda$ is the ultraviolet cutoff. Expanding Eq. (12), we obtain

$$
V(\psi) \sim\left[\frac{1}{4 \pi^{2}} \rho \cos \left(\frac{\psi}{v}\right)+O\left(M_{a b}^{4}\right)\right] \ln \frac{\Lambda^{2}}{\Lambda_{\rho}^{2}},
$$

where $\rho \equiv\left(M_{e e}+M_{\mu \mu}+M_{\tau \tau}\right)\left(M_{e \mu} M_{\mu \tau} M_{e \tau}\right), \Lambda_{\rho}^{2} \equiv M_{e e}^{2}+M_{\mu \mu}^{2}+M_{\tau \tau}^{2}+2 M_{e \mu}^{2}+2 M_{\mu \tau}^{2}+2 M_{e \tau}^{2}$ and $\psi \equiv \psi_{1}-\psi_{2}+\psi_{3}$. Note that in our minimal model, $M_{e e}$ and $M_{\mu \mu}$ are zero. We shall concentrate on the field of $\psi$, which is the linear combination of the pNGBs. We will demonstrate that in our model, the field $\psi$ plays a role of the acceleron field with the potential $V \sim O\left(m_{\nu}^{4}\right)$ and the effective mass $m_{\psi}^{2} \sim O\left(m_{\nu}^{4} / v^{2}\right)$. To do this, we first examine the case in which the energy density in the dark sector $\rho_{\text {dark }}$ is made of the densities of neutrinos $\left(\rho_{\nu}\right)$ and dark energy $\left(\rho_{\mathrm{DE}}\right)$, given by

$$
\rho_{\text {dark }}=\rho_{\nu}+\rho_{\mathrm{DE}},
$$

where the dark energy density is assumed to be a function of neutrino masses $\left(m_{\nu}\right)$, i.e., $\rho_{D E}=\rho_{\mathrm{DE}}\left(m_{\nu}\right)$. At the present time, because neutrinos are nonrelativistic, $\rho_{\nu}=m_{\nu} n_{\nu}$, where $n_{\nu}$ is the total number density of neutrinos and antineutrinos. Hence, from Eq. (14) we get

$$
\rho_{\text {dark }}=m_{\nu} n_{\nu}+\rho_{\mathrm{DE}}\left(m_{\nu}\right)
$$

Here, we have concentrated on $\rho_{\text {dark }}$ being stationary with respect to the variation of the neutrino masses, which implies that

$$
\frac{\partial \rho_{\text {dark }}}{\partial m_{\nu}}=n_{\nu}+\frac{\partial \rho_{\mathrm{DE}}\left(m_{\nu}\right)}{\partial m_{\nu}}=0 .
$$

By defining the equation of state (EoS) parameter $w$ as $w=p / \rho$, where $p$ is the total pressure of the dark sector of the universe and $\rho$ is the total energy density of it, we find [15, 17 ]

$$
w+1 \simeq \frac{m_{\nu} n_{\nu}}{\rho_{\text {dark }}} \simeq-\frac{m_{\nu}}{\rho_{\text {dark }}} \frac{\partial V_{\mathrm{DE}}\left(m_{\nu}\right)}{\partial m_{\nu}}
$$


where in deriving the second approximate equality we have used Eq. (16) and $\rho_{\mathrm{DE}}\left(m_{\nu}\right) \simeq$ $V_{\mathrm{DE}}\left(m_{\nu}\right)$ and $V_{\mathrm{DE}}\left(m_{\nu}\right)$ is the dark energy potential. We note that for the approximation in Eq. (17), we have neglected the contribution of any kinetic terms to the dark energy density $^{1}$.

We now discuss the case in which the neutrino masses depend on some scalar field $\mathcal{A}$, called the acceleron, $m_{\nu}=m_{\nu}(\mathcal{A})$. From Eq. (17) we obtain

$$
w+1 \simeq-\frac{m_{\nu}}{\rho_{\text {dark }}} \frac{\partial V_{\mathrm{DE}}\left(m_{\nu}\right)}{\partial \mathcal{A}} \frac{1}{\partial m_{\nu} /(\partial \mathcal{A})}
$$

According to the five-year WMAP data on the anisotropy of the CMB radiation [29], the observed value of $w$ is $w \simeq-1$. To have $w \simeq-1$, it follows from Eq. (18) that the potential for $\mathcal{A}$ has to be very flat, i.e., $\partial V_{\mathrm{DE}}\left(m_{\nu}\right) /(\partial \mathcal{A}) \simeq 0$, and/or the dependence of $m_{\nu}$ on $\mathcal{A}$ has to be very steep, i.e., $\partial m_{\nu} /(\partial \mathcal{A}) \gg 1$.

Hence, in our model the pNGB $\psi$ corresponds to the acceleron field $\mathcal{A}$. From Eq. (13), we find that the dark energy potential is given by

$$
V_{\mathrm{DE}}=V(\psi) \sim m_{\nu}^{4} \cos \left(\frac{\psi}{v}\right) .
$$

For $|\psi / v| \ll 1$, which can be satisfied if the scale $v$ is sufficiently large, we find that

$$
|\partial V(\psi) /(\partial \psi)|=\left(m_{\nu}^{4} / v\right) \sin (\psi / v) \simeq 0
$$

Thus, it follows from Eqs. (18) and (20) that in this model the observed relation $w \simeq-1$ can be realized.

\section{SUMMARY}

In summary, we have considered the generation of the small neutrino mass through the radiative mechanism in the extended Babu-Zee models. We have shown that the generated small neutrino masses depend on a pNGB, which can play a role of the acceleron field and the potential energy of the pNGB can be the dark energy potential. In particular, we have demonstrated that the observed value of the EoS parameter from WMAP can be realized.

\footnotetext{
1 The accuracy of this approximation is shown in Ref. [15].
} 


\section{Acknowledgments}

We would like to thank Prof. T. C. Yuan for discussions. This work is supported in part by the National Science Council of R.O.C. under Grant \#: NSC-95-2112-M-007-059-MY3 and National Tsing Hua University under Grant \#: 97N2309F1.

[1] W. M. Yao et al. (Particle Data Group), J. Phys. G 33 (2006) 1.

[2] R. N. Mohapatra et al., Rept. Prog. Phys. 70, 1757 (2007) arXiv:hep-ph/0510213.

[3] A. Zee, Phys. Lett. B93, 389 (1980) [Erratum-ibid. B 95, 461 (1980)]; Phys. Lett. B161, 141 (1985).

[4] A. Zee, Nucl. Phys. B264, 99 (1986); K.S. Babu, Phys. Lett. B203, 132 (1988).

[5] C. S. Chen, C. Q. Geng and J. N. Ng, Phys. Rev. D75, 053004 (2007); C. S. Chen, C. Q. Geng, J. N. Ng and J. M. S. Wu, JHEP 0708, 022 (2007); C. S. Chen, C. Q. Geng and D. V. Zhuridov, arXiv:0801.2011 [hep-ph].

[6] D. N. Spergel et al. [WMAP Collaboration], Astrophys. J. Suppl. 148, 175 (2003); H. V. Peiris et al. [WMAP Collaboration], ibid. 148, 213 (2003); D. N. Spergel et al. [WMAP Collaboration], ibid. 170, 377 (2007).

[7] S. Perlmutter et al. [Supernova Cosmology Project Collaboration], Astrophys. J. 517, 565 (1999); A. G. Riess et al. [Supernova Search Team Collaboration], Astron. J. 116, 1009 (1998); P. Astier et al. [The SNLS Collaboration], Astron. Astrophys. 447, 31 (2006); A. G. Riess et al., arXiv:astro-ph/0611572.

[8] For a recent review, see J. Frieman, M. Turner and D. Huterer, arXiv:0803.0982 [astro-ph].

[9] P. J. E. Peebles and B. Ratra, Rev. Mod. Phys. 75, 559 (2003); V. Sahni, AIP Conf. Proc. $\mathbf{7 8 2 , 1 6 6}(2005)$.

[10] T. Padmanabhan, Phys. Rept. 380, 235 (2003).

[11] E. J. Copeland, M. Sami and S. Tsujikawa, Int. J. Mod. Phys. D 15, 1753 (2006).

[12] R. Durrer and R. Maartens, Gen. Rel. Grav. 40, 301 (2008).

[13] S. Nojiri and S. D. Odintsov, Int. J. Geom. Meth. Mod. Phys. 4, 115 (2007) arXiv:hep-th/0601213; arXiv:0801.4843 [astro-ph].

[14] C. T. Hill, D. N. Schramm and J. N. Fry, Comments Nucl. Part. Phys. 19, 25 (1989); J. A. Frie- 
man, C. T. Hill and R. Watkins, Phys. Rev. D 46, 1226 (1992); A. K. Gupta, C. T. Hill, R. Holman and E. W. Kolb, ibid. 45, 441 (1992); J. A. Frieman, C. T. Hill, A. Stebbins and I. Waga, Phys. Rev. Lett. 75, 2077 (1995).

[15] R. Fardon, A. E. Nelson and N. Weiner, JCAP 0410, 005 (2004).

[16] P. Gu, X. Wang and X. Zhang, Phys. Rev. D 68, 087301 (2003).

[17] R. D. Peccei, Phys. Rev. D 71, 023527 (2005).

[18] R. Takahashi and M. Tanimoto, Phys. Lett. B 633, 675 (2006); R. Fardon, A. E. Nelson and N. Weiner, JHEP 0603, 042 (2006).

[19] A. W. Brookfield, C. van de Bruck, D. F. Mota and D. Tocchini-Valentini, Phys. Rev. Lett. 96, 061301 (2006); Phys. Rev. D 73, 083515 (2006) [Erratum-ibid. D 76, 049901 (2007)].

[20] C. T. Hill, I. Mocioiu, E. A. Paschos and U. Sarkar, Phys. Lett. B 651, 188 (2007).

[21] P. H. Gu, H. J. He and U. Sarkar, Phys. Lett. B 653, 419 (2007); JCAP 0711, 016 (2007).

[22] P. H. Gu, Phys. Lett. B 661, 290 (2008).

[23] J. R. Bhatt, P. H. Gu, U. Sarkar and S. K. Singh, Phys. Lett. B 663, 83 (2008).

[24] J. R. Bhatt and U. Sarkar, arXiv:0805.2482 [hep-ph].

[25] K. S. Babu and C. Macesanu, Phys. Rev. D 67, 073010 (2003).

[26] J. van der Bij and M. J. G. Veltman, Nucl. Phys. B231, 205 (1984); K. L. McDonald and B. H. J. McKellar, arXiv:hep-ph/0309270.

[27] R. Barbieri, L. J. Hall, S. J. Oliver and A. Strumia, Phys. Lett. B 625, 189 (2005).

[28] I. Aizawa and M. Yasue, Phys. Rev. D 73, 015002 (2006) arXiv:hep-ph/0510132.

[29] E. Komatsu et al. [WMAP Collaboration], arXiv:0803.0547 [astro-ph]. 